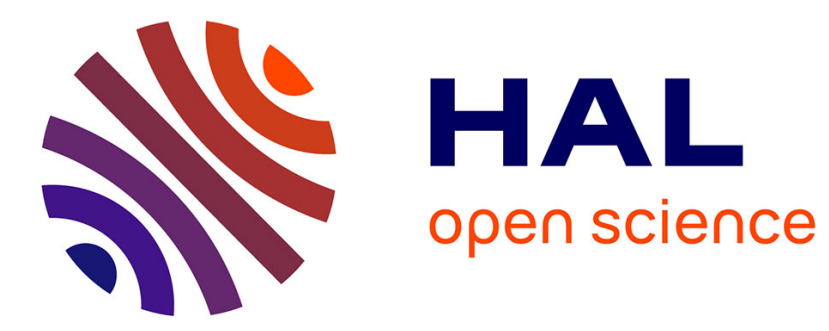

\title{
Un programme pour des territoires français innovants
}

Gérard-François Dumont

\section{To cite this version:}

Gérard-François Dumont. Un programme pour des territoires français innovants. Population et avenir, 2017, 731, pp.3. 10.3917/popav.731.0003 . hal-01501387

\section{HAL Id: hal-01501387 \\ https://hal.science/hal-01501387}

Submitted on 4 Apr 2017

HAL is a multi-disciplinary open access archive for the deposit and dissemination of scientific research documents, whether they are published or not. The documents may come from teaching and research institutions in France or abroad, or from public or private research centers.
L'archive ouverte pluridisciplinaire HAL, est destinée au dépôt et à la diffusion de documents scientifiques de niveau recherche, publiés ou non, émanant des établissements d'enseignement et de recherche français ou étrangers, des laboratoires publics ou privés. 


\section{Un programme pour des territoires français innovants}

\begin{abstract}
Le rôle des territoires pour contribuer à toutes les innovations, économiques et sociales, afin de viser le bien commun des citoyens, est essentiel. Dans ce dessein, il importe que la France inverse ses tendances à la (re)centralisation et déploie une politique pertinente d'aménagement du territoire.
\end{abstract}

\section{Revenir à une décentralisation libératrice des énergies des territoires}

La France s'est heureusement décentralisée en 1982. Il s'en est suivi une période de quinze années qui peut être qualifiée des « quinze glorieuses de la décentralisation $»^{1}$. Puis, au tournant du nouveau siècle, la France a privilégié des décisions de recentralisation sur celles permettant de libérer l'initiative dans les territoires. Cette recentralisation s'est notamment traduite par la limitation accrue de l'autonomie fiscale des collectivités territoriales². Bien entendu, les recettes « nationalisées » font théoriquement l'objet de compensations par des dotations de l'État, mais les méthodes de calcul et l'évolution de la compensation restent douteuses et aléatoires.

Quant à ce qui a été initialement appelé, en 2003, l'acte II de la décentralisation, il a d'abord fait souffler un vent d'optimisme. En effet, la réforme constitutionnelle de 2003 a complété l'article $1^{\text {er }}$ de la Constitution, désormais ainsi rédigé : « La France est une République indivisible, laïque, démocratique et sociale. [...] Son organisation est décentralisée $»^{3}$. En réalité, pour les collectivités territoriales, cet acte II s'est surtout traduit par des contraintes réglementaires sous forme de ce que je définirais une " décentralisation centralisée ». Décentralisation, puisque ce sont bien les élus des collectivités territoriales qui votent un nombre accru de décisions, mais centralisée, puisque les contraintes nationales légales qui définissent les règles de ces décisions sont d'une ampleur considérable.

Depuis, les lois territoriales ${ }^{4}$ se sont multipliées surtout pour imposer aux collectivités territoriales de nouvelles contraintes coûteuses et chronophages.

1. Dumont, Gérard-François, « Favoriser une meilleure gouvernance des territoires », dans: Allain, Joël, Goldman, Philippe, Saulnier, Jean-Pierre, De la prospective à l'action, Apors Éditions, Bourges, 2016.

2. Avec différentes mesures : suppression des recettes fiscales des collectivités territoriales correspondant à la part salaire de la taxe professionnelle, suppression des recettes des régions correspondant à la part régionale de la taxe d'habitation, suppression de la vignette des véhicules de particuliers, dont les recettes étaient auparavant affectées, depuis 1984, aux départements..

3. Loi constitutionnelle du 28 mars 2003 , « relative à l'organisation décentralisée de la République » et au référendum local.

4. Cf. par exemple Dumont, Gérard-François, « Les réformes territoriales en France, quel diagnostic ? », Fondation Res Publica, $n^{\circ}$ 93, 28 septembre 2015 ; Torre, André, Bourdin, Sébastien, «France : Des réformes territoriales qui posent bien des questions », Population \& Avenir, $n^{\circ} 727$, mars-avril 2016.
Revenir à la décentralisation implique deux impératifs : d'une part, que les lois territoriales se contentent de fixer des objectifs, sans détailler de façon uniforme les moyens de les atteindre et, d'autre part, que des compétences soient transférées efficacement dans les domaines souhaitables, notamment en supprimant les doublons.

\section{Les deux fondements d'une politique pertinente d'aménagement du territoire}

La politique implicite d'aménagement du territoire repose actuellement sur deux paradigmes erronés, équivalents à des lubies idéologiques. Le premier consiste à se figurer que la compétitivité de la France ne peut reposer que sur ses métropoles, que l'État devrait tout particulièrement favoriser. Pourtant, les analyses les plus objectives montrent que, si le processus de métropolisation peut exister, il ne déclenche pas automatiquement d'« effet métropole $»^{5}$. Le second paradigme consiste à continuer de penser selon un modèle centrepériphérie $^{6}$ alors qu'aujourd'hui, les logiques territoriales sont devenues beaucoup moins radiales que réticulaires, ce qui est d'ailleurs stimulé par les logiciels géographiques des smartphones.

La politique nationale d'aménagement du territoire doit
Il importe

que la France

inverse ses

tendances

à la (re)

centralisation

et déploie

une politique

pertinente

d'aménagement

du territoire.

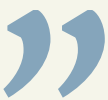
donc reposer sur deux fondements pour donner des chances d'innovation à tous les territoires. Le premier consiste à instaurer l'égalité des territoires dans les supports financiers de l'État et, par exemple, à égaliser le dotation générale de fonctionnement de base qui doit être égale par habitant, quelle que soit la taille démographique de la collectivité territoriale. Le second fondement concerne le numérique, outil impératif d'attractivité. C'est à l'État de permettre aux territoires de ne pas être pénalisés dans ce domaine car il est mieux placé que n'importe quelle collectivité territoriale pour s'imposer face aux opérateurs, compte tenu du nombre d'abonnés qu'il peut représenter.

Toute innovation territoriale ${ }^{7}$ ne s'imagine et ne se concrétise que lorsque des citoyens éprouvent un attachement à leur territoire. Et comme il n'est pas aisé de tomber amoureux d'une intercommunalité au périmètre décidé d'en haut, sans recueil de l'avis des citoyens, tout doit être mis en œuvre pour permettre le déploiement de projets territoriaux à toutes les échelles géographiques, donc également à l'échelle de base qu'est la commune. -

5. Poupard Gilles, « Développement local et emploi productif : un monopole des métropoles? », Population \& Avenir, $\mathrm{n}^{\circ} 725$, novembre-décembre 2015 ; Zaninetti, Jean-Marc, "La crise et l'attractivité des villes: Paris en recul», Population \& Avenir, $n^{\circ} 730$, novembre-décembre 2016.

6. Dumont, Gérard-François, « Territoires : un fonctionnement radial ou réticulaire? », Population \& Avenir, $n^{\circ}$ 723, mai-juin 2015.

7. Dumont, Gérard-François, « La France des marges et l'indispensable attractivité des territoires », dans : Woessner, Raymond, La France des marges, Paris, Atlande, 2016. 